\title{
Spectrum Occupancy Information in Support of Adaptive Spectrum Sensing for Cognitive Radio
}

\author{
Kishor Patil, Knud Skouby, Ramjee Prasad \\ Aalborg University \\ Denmark
}

Tel: +91-9423082407Ｅ-mail: kpp@es.aau.dk

Snehal Barge

Sinhgad Academy of Engineering, Pune University

Pune, India

Tel: +91-9665542926Ｅ-mail: snehal.barge6@gmail.com

Received: December 31, 2013 Accepted: March 13, $2014 \quad$ Published: April 30, 2014

DOI: 10.5296/npa.v6i1.4862

URL: http://dx.doi.org/10.5296/npa.v6i1.4862

\begin{abstract}
Cognitive radio (CR) is an emerging solution to spectrum scarcity currently faced worldwide as CR can change its operating parameters like frequency and bandwidth according to the radio environment and provides efficient spectrum utilization. Many measurement campaigns conducted around the world showed that the licensed spectrum is not efficiently utilized which led to the need of implementation of dynamic spectrum access (DSA). Statistical information about current frequency usage and its use for developing new algorithms and protocols for CR network is the research area that still requires much more effort. In this paper we discuss the comparison of measurement results obtained from two measurement campaigns conducted in Pune at same location but with different total bandwidths considered. Further to implement CR the best suited channels are obtained from the data collected by using adaptive sensing methods, reference, duty cycle, periodicity and Markov method. The results of spectrum sensing methods show that spectrum occupancy information play a vital role in sensing best suited channels for cognitive radio.
\end{abstract}

Keywords: Adaptive Spectrum Sensing, Cognitive Radio (CR), Dynamic Spectrum Access (DSA), Spectrum Occupancy. 


\section{Introduction}

Advance technologies are being introduced in the market but the problem we are facing is the shortage of spectrum to accommodate all the upcoming technology. The spectrum allocated to the licensed user is underutilized as concluded by many measurement campaigns conducted around the globe. One of the resourceful approaches for efficient utilization of licensed spectrum is Cognitive radio. Cognitive Radio [1], [2] is a paradigm for wireless communication in which wireless node changes its transmitting parameters according to its radio environment for efficient and reliable communication and avoiding interference with licensed or unlicensed users. The cognitive radio has ability to dynamically optimize the operating parameters like frequency and bandwidth according to RF environment variations [3]. By applying the CR technique the deficiency of the spectrum can be overcome.

The numerous measurement results [4]-[11] have showed poor spectrum utilization of the licensed spectrum. All these measurement campaigns have shown significant amount of unused spectrum both in frequency and time domain. The unused spectrum termed as white space were found despite of the fact that most of the spectrum is licensed. This has led to the conclusion that the current spectrum regulation is highly inefficient. The Dynamic Spectrum Access (DSA) vision tries to solve this problem and improve the efficiency of spectrum usage. The unlicensed users which are also termed as secondary users sense the unused spectrum bands and opportunistically use those without harmful interference to licensed users also sometimes referred as primary users.

The availability of the frequencies and further development of algorithm for efficient frequency allocation in cognitive radio network can be derived from the statistical information of frequency usage in the primary network. One of the contributions of this paper is to present the current spectrum usage of GSM band using the real time measurements performed at Pune, India. The frequency band considered for this work are $700-2746.6 \mathrm{MHz}$ and GSM 900 uplink band (890-915MHz).

To improve the efficiency of selecting a suitable channel for CR different sensing methods are used [12]. Spectrum sensing method opts for an appropriate channel among other pre-determined channels in the band. Merits of spectrum sensing are that there is decrease in the time as well as power consumption because larger the bandwidth to be sensed more is the power consumed. Another contribution of this paper is comparison of four spectrum sensing methods, reference case, duty cycle, periodicity and Markov methods on the extensive data collected during measurement campaign. This paper is the extended version of our previous paper [13] in which we have presented measurement results of GSM band.

The remainder of this paper is structured as follows. Section II describes measurement setup and methodology. Section III gives the comparison of the result obtained from the measurements taken. Suitable data is considered form the results obtained from the comparison and are further used for adaptive sensing which are explained in section IV. The results of adaptive spectrum sensing are discussed in section $\mathrm{V}$ while the conclusion drawn from the results are discussed in section VI. 


\section{Measurement Setup}

The measurement setup shown in figure 1 consists of spectrum analyzer, a laptop and an antenna. We used a Rohde and Schwarz (FSH3) spectrum analyzer in order to take measurements. The parameter settings are shown in Table 1. It was interfaced with laptop via an optical cable. The spectrum analyzer was programmed to save real-time information about the spectrum in the laptop's hard disk. AOR DA 5000 antenna is used in this campaign. It is specially designed for the frequency range of $700 \mathrm{MHz}$ to $3 \mathrm{GHz}$. The antenna is connected to spectrum analyzer through a low loss coaxial cable. The details of measurement set up, methodology and spectrum analyzer configuration are specified in [11]. The setup was installed on the terrace of a commercial building on law college road which is one of the busy locations in Pune. The area is surrounded by residential buildings, hotels and restaurants. For data collection, a program is written in the Remote Control software of FSH using the commands for spectrum analyzer. The program is executed for the number of times as per the count given in loop. According to the period of measurement, we set the loop such that data collection goes on continuously without interruption. The files are collected in .CSV format. These files can be opened in Microsoft excel. Then the data is processed and viewed for analysis and comparison. The detail descriptions of the parameters set for measurement are as below. The stored data is processed using the MATLAB software package, and the data is converted to a more understandable format for future analysis.

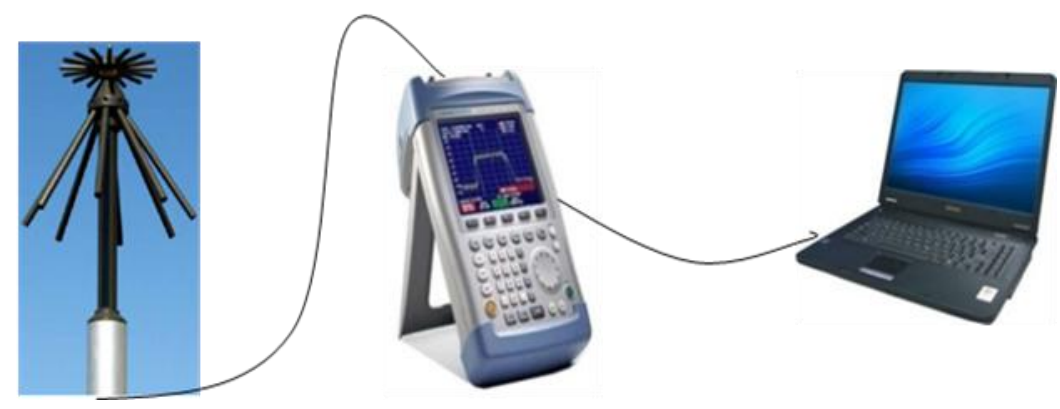

Figure 1. Measurement Setup.

\begin{tabular}{|c|c|c|}
\hline Parameters & Setting 1 & Setting 2 \\
\hline \hline Frequency range & $890-950 \mathrm{MHz}$ & $700-2746.6 \mathrm{MHz}$ \\
\hline Frequency span & $60 \mathrm{MHz}$ & $60 \mathrm{MHz}$ \\
\hline Resolution BW & $100 \mathrm{KHz}$ & $100 \mathrm{KHz}$ \\
\hline Number of measurement points & 301 & 10234 \\
\hline Sweep time & $2.25 \mathrm{sec}$ & $-20 \mathrm{dBm}$ \\
\hline Reference level & $-20 \mathrm{dBm}$ & RMS \\
\hline Trace detector & RMS & 12 hours for 2 days \\
\hline Duration of measurements & 12 hours for 2 days & \\
\hline
\end{tabular}

Table 1. Parameter Setting of Spectrum Analyzer.

We report about two measurement campaigns conducted at above mentioned location with different frequency range under measurement. The first measurement for frequency band 700-2746.6 MHz is well described in [11]. The second measurement campaign was 
conducted at same location for specific GSM mobile services in GSM 900 band during 10 AM to 10 PM for four days to study the opportunities of secondary use in GSM band. We got approximately 19177 measurement traces for each day.

To distinguish noise from active primary signal threshold is necessary. Threshold setting is considered to be the most crucial step in evaluation of spectrum occupancy. For spectrum occupancy measurement methods we adopted fixed threshold which usually distinguish signal from noise. Once the power level exceeds a threshold, it will be considered as active signal, otherwise as noise. We have obtained the average noise level by connecting a $50 \Omega$ termination to spectrum analyzer. The decision threshold is set by adding $3 \mathrm{~dB}$ in measured thermal noise. The decision threshold obtained with this method is not constant since the system noise slightly increases with the frequency.

\section{Comparison of Measurement Results}

This section presents comparison of the results of GSM 900 uplink band obtained from the data collected from two measurement campaigns. We focus on the precision of results obtained from the measurement campaigns to conclude which measurement scenario gives more accurate results which can be used further for adaptive spectrum sensing.

The GSM uplink band was selected from the complete band (700-1746.6 MHz) for the comparison with the data collected with setting 1. The GSM uplink band has shown cognitive radio potentials from the measurement results obtained [13]. The results obtained are described in next section.

\subsection{Occupancy}

The computation of occupancy gives an estimation of percentage utilization of the band during the measurement period of time. The occupancy of the band is calculated by number of points above threshold divide by total number of points during the measurement period. The average threshold considered is $-90 \mathrm{dBm}$. The occupancy obtained for setting 1 (only GSM uplink band) is $21.75 \%$ while occupancy obtained for setting 2 (whole band) is $3.8917 \%$.

The big difference in occupancy even being on the same location and for same duration can be explained due to major time difference between the sweep times of the settings. In the setting 2 it takes 1.5 minutes for the next sweep to arrive. This duration is long enough to skip primary users activity in between due to which the occupancy obtained less. The occupancy results show that the setting 1 is more precise than setting 2 since it takes only 2.25 seconds to scan $890-950 \mathrm{MHz}$ band.

\subsection{Power Spectral Density (PSD) Plot}

In order to give a general overview of the spectrum usage of GSM uplink band we show average power spectral density (PSD) measured per setting at the measurement location. The PSD plots of GSM uplink band for both settings are shown in figure 2 and 3. Figure 2 show more primary user activity above decision threshold than figure 3 . This shows the fast scanning of the frequency range of measurement gives better results. 


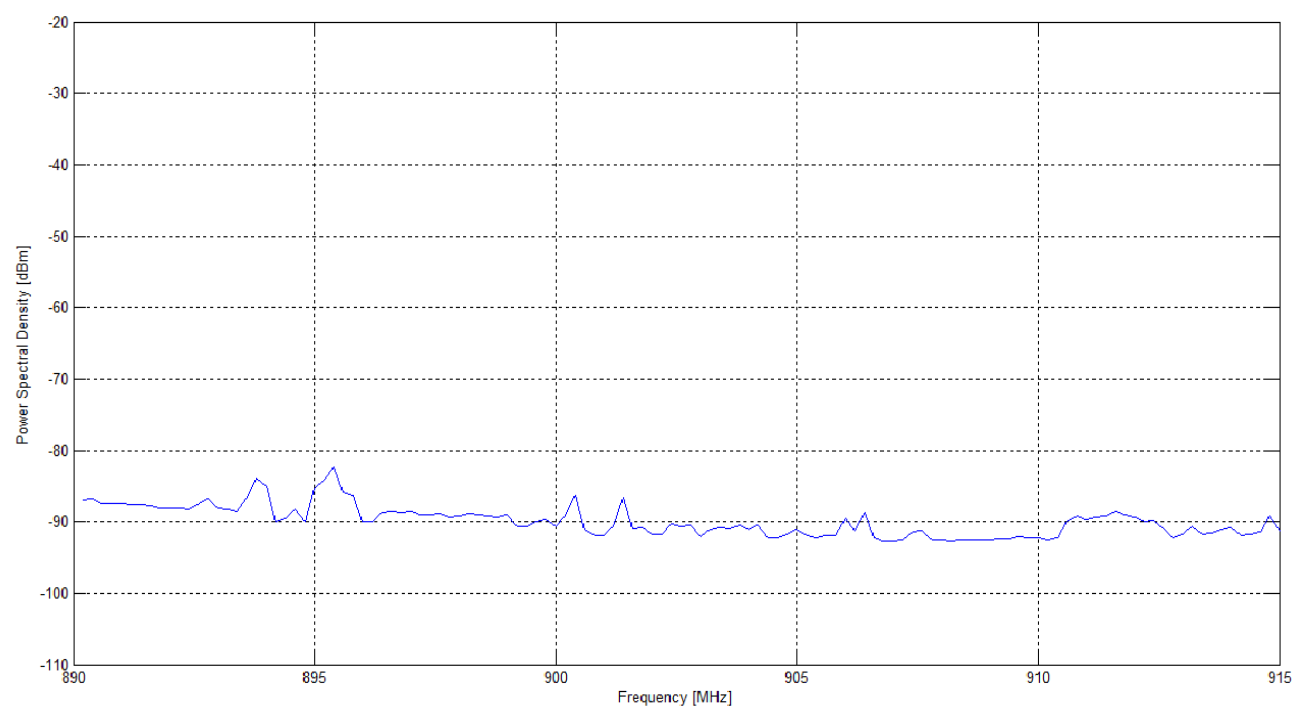

Figure 2. Average PSD plot of GSM band for setting 1.

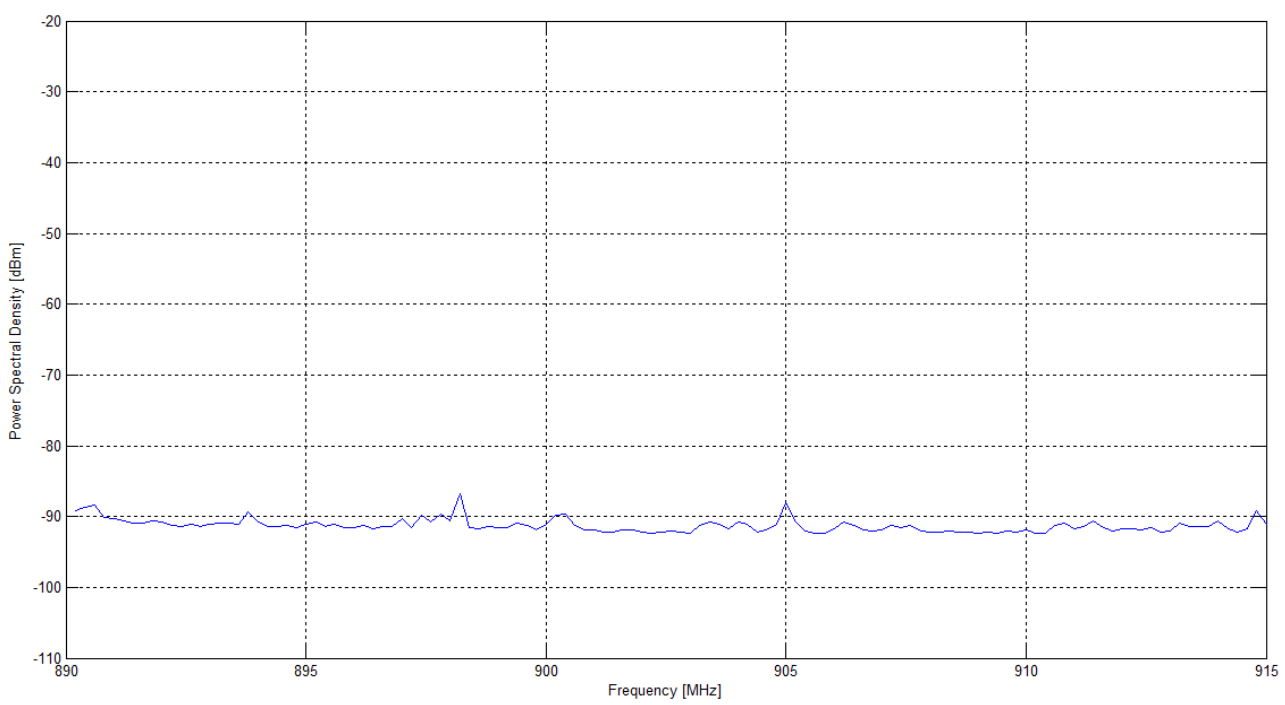

Figure 3. PSD plot of GSM band for setting 2.

\subsection{D Histogram}

3D histogram illustrates three dimensional view where the three axes represents frequency, time and power level.

Figure 4 and 5 shows the 3D histogram of GSM band for both the settings of spectrum analyzer for which the measurement were taken. Figure 4 and 5 shows the variations PSD of the uplink signal with frequency and time. As can be seen the figure 4 provides more accurate frequency occupancy with respective to time than figure 5. The GSM uplink band signals are stronger and dense in Figure 4. From the figures it is proved that the measurement data obtained by using the setting 1 for GSM uplink band are more accurate than setting 2 . 


\section{Ml Macrothink}

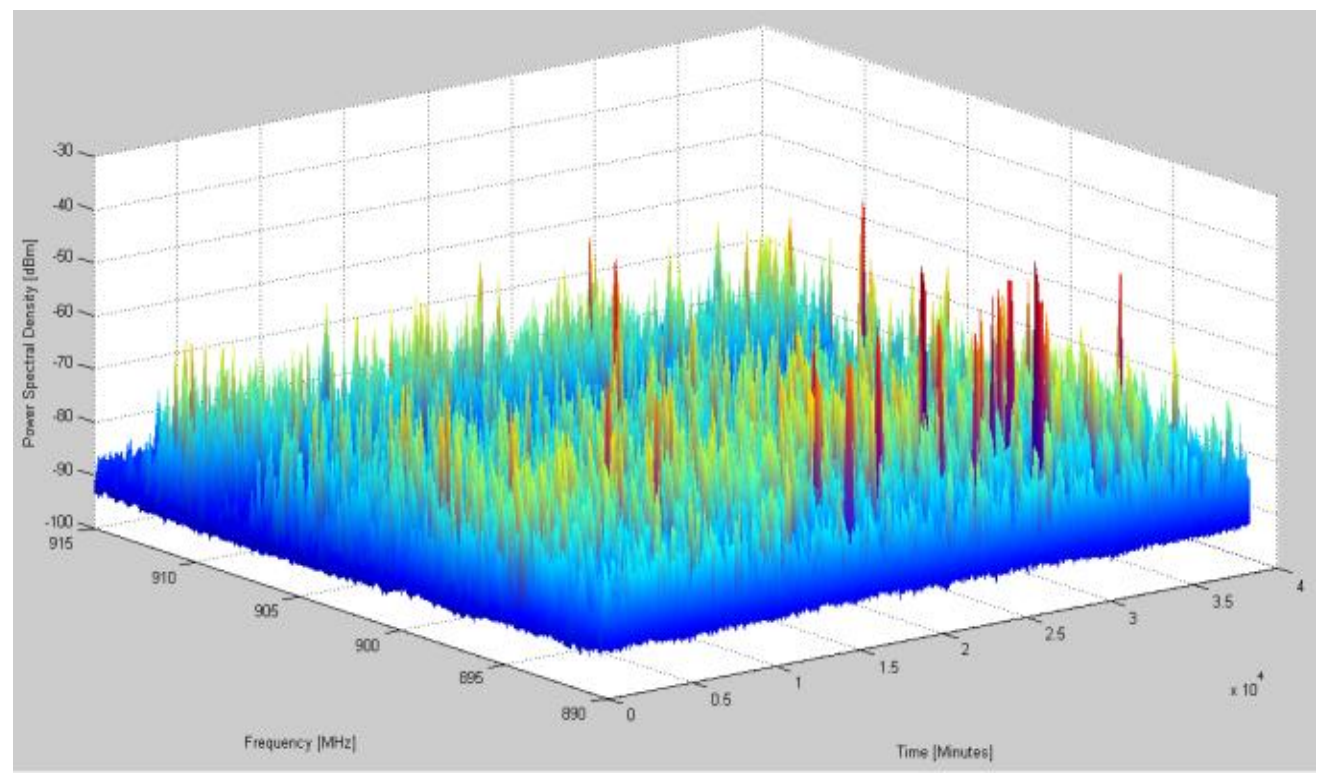

Figure 4. 3D Histogram of GSM band for setting 1.

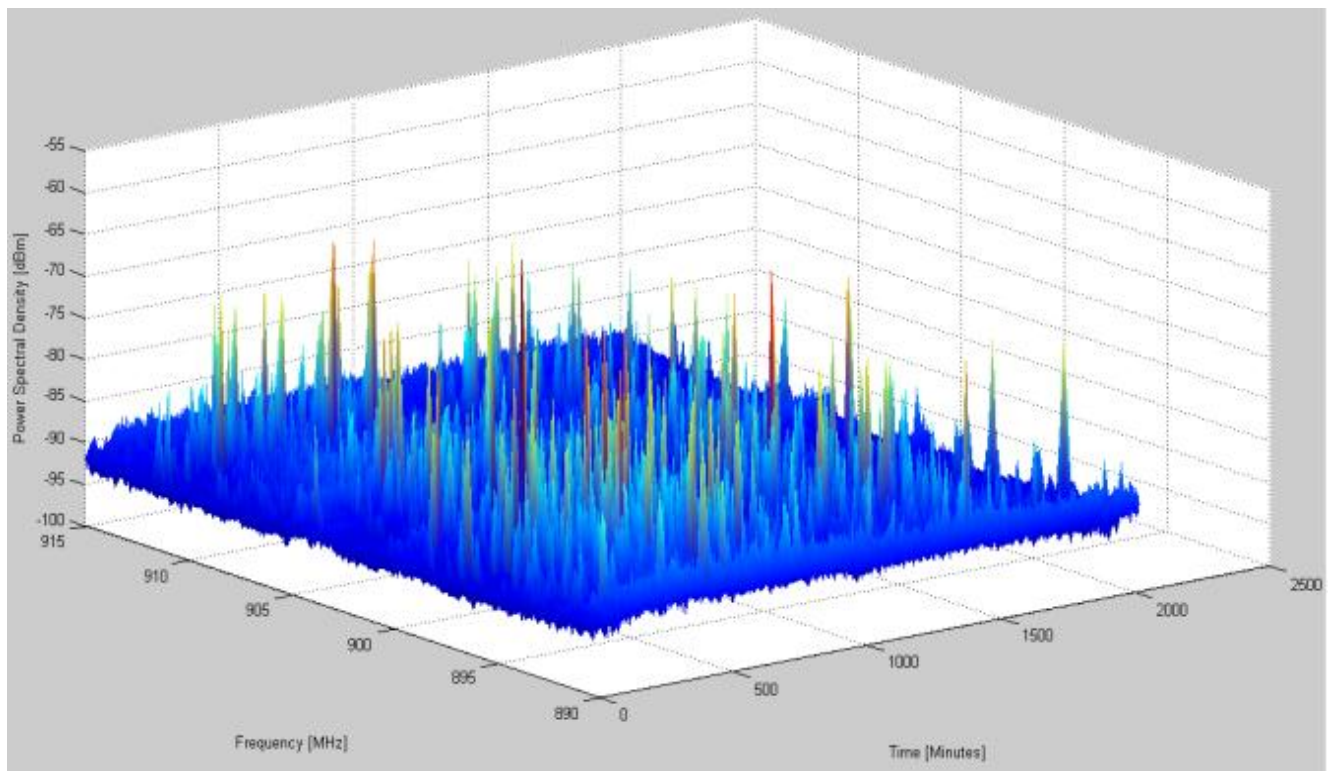

Figure 5. 3D Histogram of GSM band for setting 2.

From above all the obtained results it has been verified that the results obtained by considering only GSM uplink band are more accurate than considering the whole band. The reason behind the accuracy is that it takes maximum 2.25 seconds for the next sweep to arrive thus increases the number of files collected per minute. In total measurement period more number of files is collected than obtained by considering the complete band and thus capturing even the diminutive variations in the signal with time. So we have used data obtained of GSM uplink band from setting 1 for adaptive spectrum sensing. 


\section{Adaptive Spectrum Sensing}

Adaptive spectrum sensing is valuable because it helps to minimize the amount of spectrum to be sensed because the spectrum that was previously occupied is more likely occupied at present [14]. Spectrum sensing in the range of gigahertz will consume lot of time and power which is drawback for mobile devices. Therefore, a good policy for adaptive spectrum sensing is to identify channels with the high probability of being free. Mathias et al has introduced four sensing method in [12] which are based on a set of probabilities that describe a binary occupancy state machine for each single channel by using historical information. These methods are described in next section. These methods are applied to the data collected. We have evaluated approximately 38000 measurement traces evaluated in order to determine the respective probabilities for each channel.

\subsection{Reference Case}

This technique does not use any past information of channel occupancy to select the channel and hence there is less probability of a channel selected being free. The selection of channel for sensing is randomly done. The probability of selecting a channel which will be free is thus $P_{R R F}($ free $)=\mathbb{E}\left(1-D C_{i}\right)$ where $P_{R R F}$ (free) is the probability that the channel selected by the reference method will be free, $D C_{i}$ is the duty cycle as measured at channel $i$ and $\mathbb{E}\left(1-D C_{i}\right)$ is one minus the average duty cycle over all channels.

\subsection{Duty Cycle based Method}

Duty cycle method is based on past information about channel occupancy to select the channel and hence there is high probability of a channel selected being free. Duty cycle method always selects the lowest duty cycle channel as shown in equation (1).

$$
P_{D C}(\text { free })=1-\min \left(D C_{i}\right)
$$

$P_{D C}$ (free) is probability of channel selected by duty cycle based method will be free and $\mathrm{DC}_{i}$ is the duty cycle of channel $i$.

\subsection{First Order Markov Chain}

Parameter settings for spectrum analyzer of sweep time is set to automatic which gives a inter sweep time of 1 second. Another 1 second for the data to be transferred from spectrum analyzer to the laptop, giving us the total inter sample time of 2 second. We assumed that most connections last longer than such short period. Thus, the probability to stay in the current state will be much higher than that the channel state switches between state free and state occupied. Since, we are implementing first order markov chain we consider the state of previous sample only. We determine the probability $\alpha=P_{\text {MARKoV }}\left(\right.$ occupied $_{\left.\mid \text {free }_{\mathrm{t}-1}\right)}$ ) to switch from state free at time index $\mathrm{t}$ - 1 to state occupied at time index $\mathrm{t}$ from our measurement trace. Later, we combine it duty cycle information to $\alpha=P_{\text {MARKOV }}\left(\right.$ free $_{t} \mid$ occupied $_{t-1}=$ $\alpha .(1-\mathrm{DC}) / \mathrm{DC}$

The probability $P_{\text {MARKOV }}($ free, $t \mid \mathrm{t}-1$, i) of a channel $i$ to be free at the time index $t$ now depend on the former state of the channel and given by equation (2). 


$$
P_{\text {MARKOV }}(\text { free, } t \mid \mathrm{t}-1, \mathrm{i})=\left\{\begin{array}{cl}
1-\alpha_{\mathrm{i}} & \text { if } \text { state }_{i}(t-1) \text { is free } \\
\alpha_{\mathrm{i}} \cdot\left(1-D C_{i}\right) / D C_{i} & \text { if state } \\
\text { if }(t-1) \text { is occupied }
\end{array}\right.
$$

Hence, we selected the best channel at each time index and average over all considered time indices ( 8050 samples $\approx$ first five hours of our measurement data) using equation (3).

$$
P_{\text {MARKOV }}(\text { free })=\mathbb{E}_{t}\left[\max _{i}\left(P_{\text {MARKOV }}(\text { free }, t \mid t-1, i)\right]\right.
$$

\subsection{Method Exploiting Periodicity}

We calculated periodicity for all the channel frequencies in the GSM uplink band. This showed that shorter periods were rarely detected during our measurement duration. We appraise this information so that it can benefit for adapting sensing. Periodicity method and Markov method are similar with an exception that a time shift of $\gamma$ sample duration is considered instead of using previous sample. We determine $\gamma$ as period below fifteen minutes. If no such period is found for few frequencies, the probability for these frequencies is based on the duty cycle method.

\section{Results}

The abovementioned four methods determine the probability that channel of $200 \mathrm{kHz}$ and above bandwidth will be free at the next time lag. There are two different cases considered for the required bandwidth sensing, consecutive and non consecutive bandwidth. In the first case the required bandwidth doesn't have to be consecutive so that found free channels can be used by different transmissions. The second case senses the bandwidth such that its consecutively serviceable so single carrier could also benefit from it.

The GSM uplink band is allocated to 890- $915 \mathrm{MHz}$ in India. Figure 6 shows the case of non consecutive bandwidth. We estimated the probability of finding the complete uplink band of $25 \mathrm{MHz}$ free when exactly same amount of bandwidth is sensed.

The reference case method graph shows probability 1 for $200 \mathrm{KHz}$ (single channel) and goes along till approximately $1 \mathrm{MHz}$ and then decreases as per requested bandwidth until it meets zero. This was rather high as reference case doesn't use any historical information for spectrum sensing. One of the reasons for such unexpected result is due to the low average occupancy.

Further the performance of sensing increases by duty cycle method which uses the historical data collected during the measurements. Figure 6 explain if historical information is used the probability of finding free spectrum can considerably be increased. The probability is 1 till approximately $16 \mathrm{MHz}$ which is high even being a licensed user. The DC method graph further decreases gradually until it reaches zero at $21 \mathrm{MHz}$. The periodicity graph isn't distinguishable with duty cycle method, as there were no short periodicities found. A Markov method gives a better performance. The graph of Markov method meets zero at approximately $23 \mathrm{MHz}$.

Figure 7 brings to our attention that reference case for consecutive bandwidth shows higher probability only at the beginning of the graph later there is a drastic fall till $5 \mathrm{MHz}$. After $5 \mathrm{MHz}$ it almost remains constant and then becomes zero at $15 \mathrm{MHz}$. The use of duty 


\section{Macrothink}

cycle method slightly affects the performance of the spectrum sensing. The highest probability i.e. 1 is shown till $4 \mathrm{MHz}$ even by duty cycle method. As discussed earlier due to low periodicities duty cycle and periodicity method cannot be clearly distinguished. Markov method operates vaguely superior than the duty cycle method.

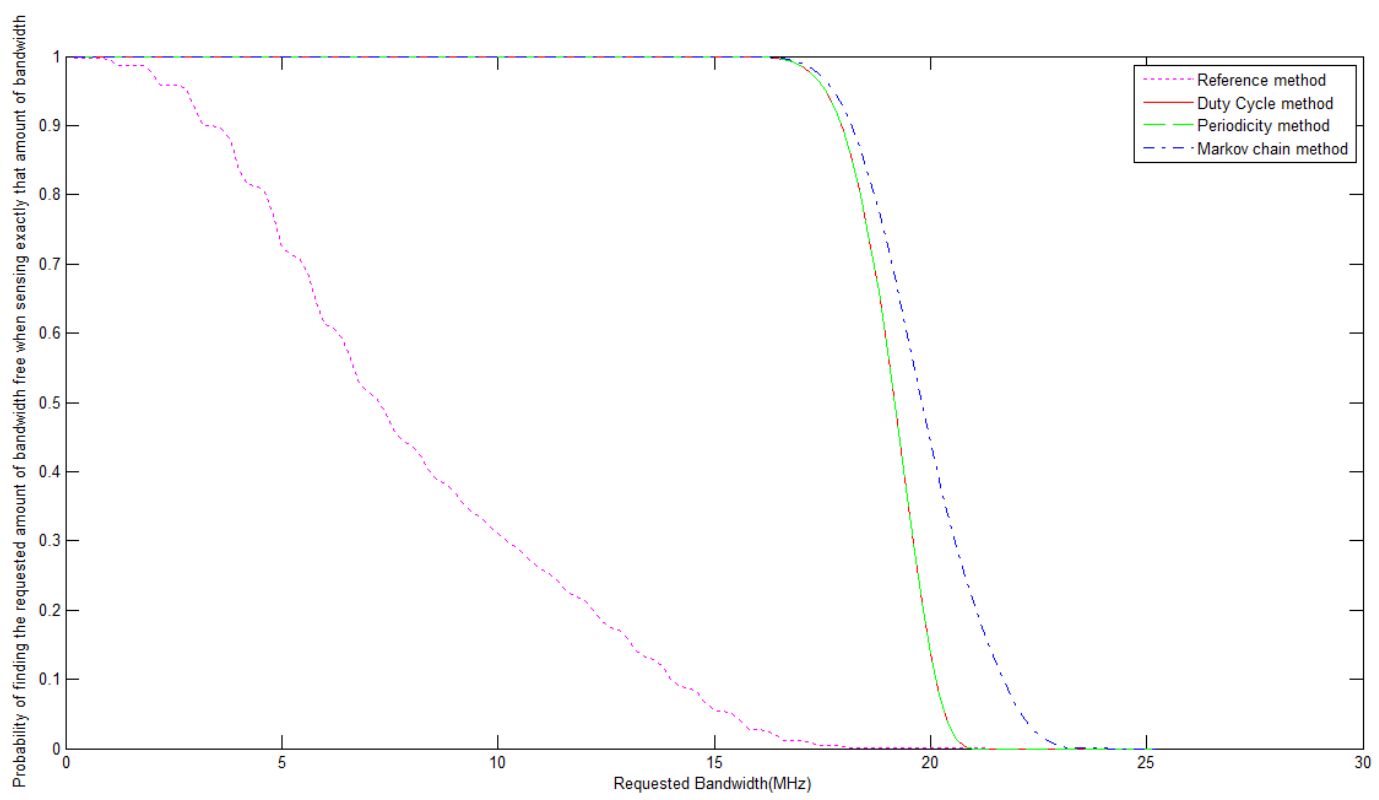

Figure 6. Comparison of the two methods for adaptive spectrum sensing for GSM uplink band for non consecutive bandwidth.

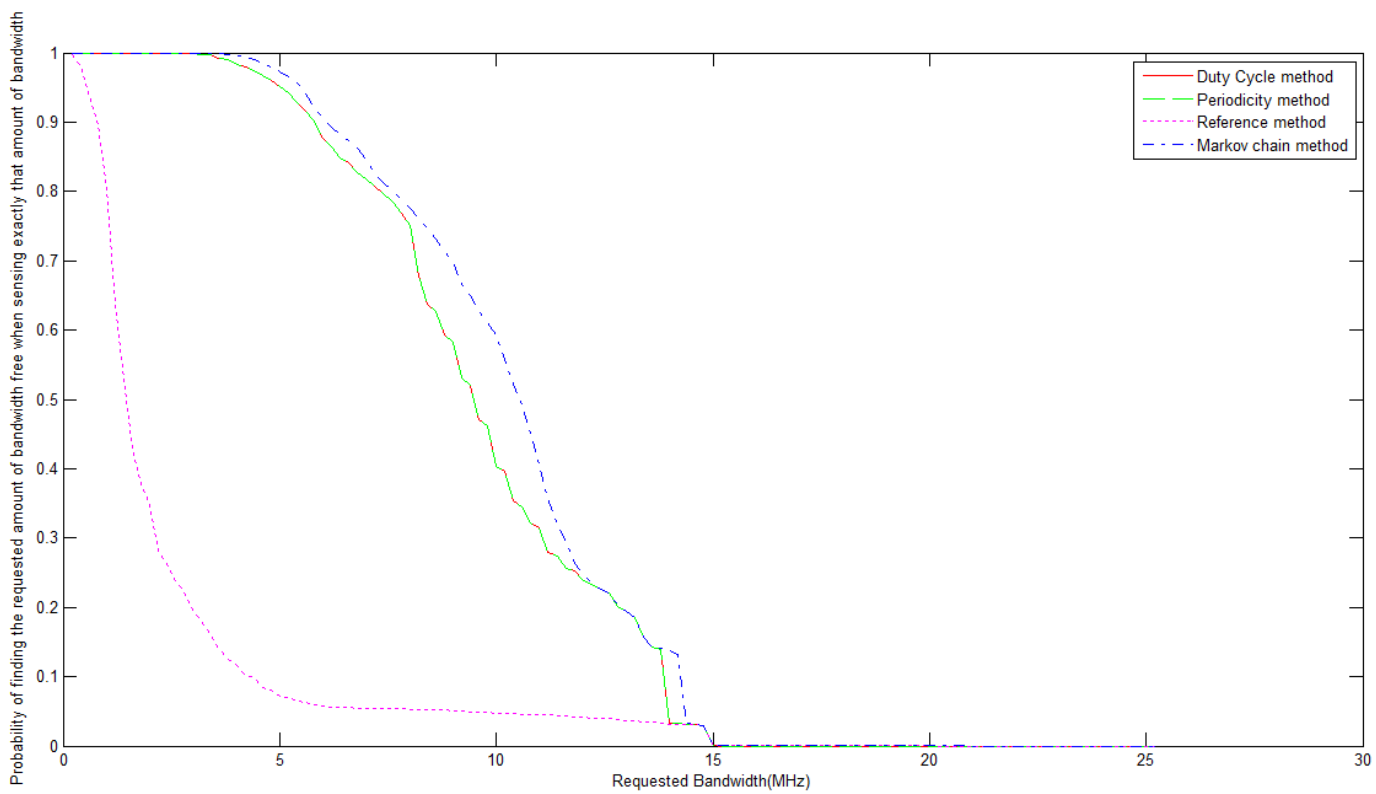

Figure 7. Comparison of the two methods for adaptive spectrum sensing for the GSM uplink band for consecutive bandwidth.

\section{Conclusion}

In this paper we reported about the accuracy of the data obtained via two different setting 
and results obtained for the adaptive sensing techniques implemented on more suitable data. The measurements carried out proved accurate results are obtained when the time lag between the trace is minimum and the number of sweep per second are greater. Due to this even the small variations of the signal can be captured which lead to more accurate results.

We evaluated the occupancy of GSM uplink band which is $21.75 \%$ for $-90 \mathrm{dBm}$ threshold. It means $75.25 \%$ of the licensed GSM uplink band is underutilized showing scope for Cognitive Radio operation. We also presented PSD and 3D histogram to show the variation of signal strength with time. We described an approach how to determine the most dominant periodicities in such spectrum usage data and showed that rarely periods shorter than five to ten minutes cannot be found.

In this paper we reported about the analysis of adaptive sensing techniques for GSM 900 band. We found the duty cycle and Markov based model performs well as compared to reference. Adaptive sensing based on historical information is advantages as it increases the probability that bandwidth selected free compared to random sensing also it is less time and power consuming.

\section{References}

[1] J. Mitola III, "Cognitive radio: an integrated agent architecture for software defined radio," Ph.D. Thesis, KTH (Royal Institute of Technology), 2000.

[2] Simon Haykin, Life Fellow, IEEE. "Cognitive Radio: Brain-Empowered Wireless Communications," IEEE Journal on Selected Areas in Communication, vol. 23, no. 2, February 2005.

http://dx.doi.org/10.1109/JSAC.2004.839380

[3] R.W. Brodersen, A. Wolisz, D. Cabric, S.M. Mishra, D. Willkomm, Corvus: a cognitive radio approach for usage of virtual unlicensed spectrum, Berkeley Wireless Research Center (BWRC) White paper, 2004.

http://dx.doi.org/ 10.1109/MICC.2009.5431523

[4] M. Wellens, J. Wu, and P. Mähönen, "Evaluation of spectrum occupancy in indoor and outdoor scenario in the context of Cognitive Radio," in Proc. of International Conference on Cognitive Radio Oriented Wireless Networks and Communications (CROWNCOM), Orlando, FL, USA, August 2007, pp. 420-427.

http://dx.doi.org/10.1109/CROWNCOM.2007.4549835

[5] Mark A. McHenry, "NSF Spectrum Occupancy Measurements Project Summary," Shared Spectrum Company Published August, 2005

[6] Zhe Wang and Sana Salous, "Spectrum Occupancy Analysis for Cognitive Radio," London Communications Symposium, University College London, 2006.

[7] Dennis A. Roberson and Cynthia S. Hood and Joseph L. LoCicero and John T. MacDonald, "Spectral Occupancy and Interference Studies in support of Cognitive Radio Technology Deployment" in Proceedings of the 1st IEEE Workshop on Networking Technologies for Software Defined Radio Networks (SDR'06), 25-25 Sept. 2006, pp. 26-35. http://dx.doi.org/10.1109/SDR.2006.4286323

[8] Lopez-Benitez, M.; Umbert, A.; Casadevall, F.; "Evaluation of Spectrum Occupancy in 


\section{Macrothink}

Network Protocols and Algorithms

ISSN $1943-3581$

2014, Vol. 6, No. 1

Spain for Cognitive Radio Applications," in Proc.of Vehicular Technology Conference, VTC Spring, 26-29 April 2009, pp. 1-5.

http://dx.doi.org/10.1109/VETECS.2009.5073544

[9] M. Islam, G. L. Tan, F. Chin, B. E. Toh, Y.-C. Liang, C. Wang, Y. Y. Lai, X. Qing, S. W. Oh, C. L. Koh, and W. Toh, "Spectrum Survey in Singapore: Occupancy Measurements and Analyses," in Proc. of International Conference on Cognitive Radio Oriented Wireless Networks and Communications (CROWNCOM), Singapore, May 2008, pp. 1-7.

http://dx.doi.org/10.1109/CROWNCOM.2008.4562457

[10]Kishor Patil, Knud Skouby, Ashok Chandra, Ramjee Prasad, "Spectrum Occupancy Statistics in the Context of Cognitive Radio," in Proc. of 14th International Symposium on Wireless Personal Multimedia Communications (WPMC'11),Brest, France, November 2011

[11]Kishor Patil, Ramjee Prasad, Knud Skouby, "Stochastic Duty Cycle Model Based on Measurement for Cognitive Radio," in Proc. of 15th International Symposium on Wireless Personal Multimedia Communications (WPMC'12), in Taipei, Taiwan, September 2012 [12] Matthias Wellens, Alexandre de Baynast and Petri Mahonen, "Exploiting Historical Spectrum Occupancy Information for Adaptive Spectrum Sensing," Department of Wireless Networks, RWTH Aachen University, Germany.

http://dx.doi.org/10.1109/WCNC.2008.132

[13] Kishor Patil, Snehal Barge, Ramjee Prasad, Knud Skouby, "Evaluation of Spectrum Usage for GSM band in Indoor and Outdoor Scenario for Dynamic Spectrum Access", in Proc. of International Conference on Advances in Computing, Communications and Informatics (ICACCI-2013), Mysore, India, August 2013.

http://dx.doi.org/10.1109/ICACCI.2013.6637250

[14]Zhao and A. Swami, "A decision-theoretic framework for opportunistic spectrum access," IEEE Wireless Communications, vol. 14, no. 4, pp. 14-20, August 2007.

http://dx.doi.org/10.1109/MWC.2007.4300978

\section{Copyright Disclaimer}

Copyright reserved by the author(s).

This article is an open-access article distributed under the terms and conditions of the Creative Commons Attribution license (http://creativecommons.org/licenses/by/3.0/). 